\title{
Stratégies de l'interviewé pour répondre à une question médiée intrusive
}

\author{
Aleksandra Nowakowska \\ Université Montpellier 3 \& Praxiling \\ aleksandra.nowakowska@univ-montp3.fr
}

Notre communication s'intéressera à l'acte réactif dialogal de la réponse de l'interviewé politique à un certain type de question, fréquent, que nous définirons en termes de question médiée, dans l'interview politique, et plus précisément aux stratégies discursives employées, afin d'éviter une réponse franche et directe qui pourrait exposer sa face, sur la scène médiatique. Le genre interview politique peut très grossièrement se définir comme relevant de l'hypergenre des interactions verbales, qu'il spécifie de la façon suivante : l'interaction s'organise entre deux parties :

— le (ou les) intervieweur(s) : un journaliste, dont le rôle est de poser des questions ;

— le (ou les) interviewé(s) : une personnalité politique, dont le rôle est de répondre aux questions qui lui sont posées.

L'interview est centrée sur la parole de l'interviewé, ses prises de position etc, devant lesquelles l'intervieweur doit s'effacer, il doit n'en être qu'un pur médiateur. Le journaliste intervieweur est là pour faire parler l'invité politique, pour tenter de faire sortir le renard de sa tanière, en questionnant sur ce qui fait problème. Les questions médiées se signalent par deux traits : (i) elles manifestent fréquemment une médiation énonciative, à savoir qu'elles se présentent comme rapportant, de différentes manières, un propos tenu antérieurement par un autre locuteur, qui appartient le plus souvent à la sphère politique ou syndicale ; (ii) ce propos est presque toujours contrediscursif, voire franchement polémique, ce qui donne à la question un tour offensif. La convocation d'autres discours permet au journaliste (i) d'être le « porteparole ", le locuteur, le relais de questions qui se posent; et (ii) de poser des questions offensives, sinon offensantes, en s'abritant derrière la médiation de la parole rapportée. Nous appellerons questions médiées toutes celles qui s'appuient de différentes manières sur le discours rapporté. La structure de la question médiée peut être décrite (en appui sur Nowakowska et Bres 2009), selon un module à 4 éléments:

(i) thème général (avec parfois appui sur le dire immédiat de l'interviewé)

(ii) jugement (offert à la mise en débat de la verbalisation de l'intervieweur)

(iii) médiation énonciative de ce jugement

(iv) interrogation peut porter directement sur le jugement : "est-ce que ii” ou se développer comme demande d'évaluation de ce jugement

Soit l'exemple suivant :

(1) Interview de J.-F. Copé par O. Galzi, dans Les quatre vérités, le 9 décembre 2008

OG29 - dernière question Xavier Bertrand va prendre la tête de l'UMP / vous êtes le chef de groupe UMP à l'assemblée (i) / on dit de lui (iii) que c'est votre meilleur ennemi (ii)// c'est vrai ? (iv)

La forme de la question médiée de l'intervieweur correspond, dans ce cas, à la structure : thème (i), jugement rapporté médié (iii+ii) + interrogation (iv). 
Si dans l'exemple analysé l'intervention de l'intervieweur réalise une interrogation explicite, du point de vue morpho-syntaxique, nous distinguerons, en appui sur C. Kerbrat (1991 et 1996), entre interrogation et question. Cette dernière correspond à un acte de langage qui peut se réaliser de différentes façons, plus au moins explicites. L'interrogation est la structure syntaxique prototypique de la question. À l'exception des rectifications ou des précisions, presque toutes les interventions de l'intervieweur sont des questions, qui ne se réalisent pas forcément de façon prototypique, par l'interrogation syntaxique.

Le fonctionnement de la médiation énonciative est contrediscursif : il s'agit le plus souvent de rapporter un discours qui contredit le discours de l'interviewé, constitue un problème, voire un piège pour lui. Ce qui se note notamment au niveau de l'identité des énonciateurs convoqués : principalement (i) les opposants politiques (médiation exogroupale), (ii) des membres d'un même bord politique, mais qui tiennent un discours différent ou contraire, parce qu'ils sont, par exemple, en concurrence (médiation endogroupale) et (iii) plus rarement, le propre discours de l'interviewé.

La question en s'appuyant sur la médiation est embarrassante pour l'interviewé qui va y répondre de différentes façons. Notre étude des stratégies employées pour répondre à une question médiée intrusive portera sur un corpus de 8 interviews politiques brèves ( 8 minutes environs chacune), diffusées à la télévision dans l'émission Les quatre vérités sur France 2, ou dans les journaux télévisés, ou filmées à la radio et diffusées en ligne, dans RTL matin, de septembre 2008 à juillet 2009. Le choix des données a été motivé par deux facteurs : (i) le souhait d'avoir les données audio-visuelles pour pouvoir à terme traiter l'aspect visuel, en prenant en considération la mimo-gestualité, afin d'étudier notamment le rapport entre la parole et la posture corporelle dans la construction de l'image du politique et (ii) la représentativité du genre : toutes les émissions étudiées sont quotidiennes et diffusées dans les créneaux horaires proches et prisés, le matin, ont plus ou moins la même durée et offrent la possibilité d'avoir l'intervention de différents journalistes ; une seule occurrence - l'interview de F.Fillon - apparaît dans un format différent, le JT du soir. Le format de l'interview politique brève et quotidienne est actuellement en fort développement dans les médias français. Ce type d'interview présente des caractéristiques formelles propres notamment par rapport à l'interview présidentielle (Clayman \& Heritage, 2002), et plus encore par rapport au débat politique (Burger 2004, de Chanay et Kerbrat 2007). Ce format correspond à l'exigence de captation qui conduit les médias à proposer des émissions susceptibles d'accrocher l'attention du récepteur et de l'empêcher de zapper. Il oblige, par contre, le journaliste à bien cibler les questions et le place fréquemment, lors de l'interview, devant l'alternative : soit poser toutes les questions qu'il avait préparées, même si les réponses ne sont pas satisfaisantes, soit, en cas d'une réponse insuffisante, reformuler la question afin d'essayer d'obtenir la réponse, quitte à réduire le nombre total des questions et sélectionner les plus pertinentes parmi toutes celles qui devaient être posées.

L'analyse des réponses permettra de construire une typologie des stratégies dans l'objectif de :

(i) catégoriser les différentes manières d'interagir avec la médiation énonciative dans la question: montrer les différentes manières dont l'homme politique exploite la médiation énonciative à des fins argumentatives, afin d'en tirer partie alors même qu'elle est censée le mettre en difficulté, ce qui sera développé dans cette communication.

(ii) comparer, dans un autre travail, les réponses des politiques aux questions sans médiation énonciative, afin d'étudier si les stratégies employées sont proches ou différentes, ce qui permettrait éventuellement d'élargir cette typologie.

(iii) étudier ultérieurement la façon dont les acteurs politiques construisent leur discours et leur image, la manière dont le statut et la crédibilité des participants sont créés dans et par le discours.

Nous distinguerons et analyserons six types de réponses. Dans le premier, l'interviewé ne tient pas compte de la médiation énonciative. Dans les cinq autres, il développe, à partir de cette médiation, une stratégie qui peut consister 1) en une invalidation de l'énonciateur rapporté ; 2) en une rectification du propos rapporté ; 3) en un recadrage de l'interaction rapportée ; 4) en un recadrage de la question suscitée par le jugement médié ; et 5) en une exploitation du contenu implicite de la question. 


\section{Ignorer la médiation embarrassante}

La stratégie qui consiste à ne pas tenir compte de la médiation énonciative, à faire comme si elle n'était pas présente dans la question, est un cas typique de la langue de bois :

(2) Interview de Luc Chatel par Jean-Michel Aphatie sur RTL, le 29 juin 2009

JMA3 - parce qu'il n'y a plus de sous dans les caisses ? Parce que la France est en faillite ? dit Libération

LCH4 - non / vous savez le gouvernement est sur trois fronts au même temps / le premier front / c'est la riposte à la crise/ ça c'est le plan de relance (...) / le deuxième front euh c'est ::: la réponse (2) / c'est la (2) maitrise du déficit / et la poursuite des reformes/ Alain Duhamel l'a dit tout à l'heure / c'est pas parce qu'il y a la crise qu'il faut pas continuer à réformer le pays (...) / le troisième front / c'est celui d'investissement dans les secteurs d'avenir (...) / la France doit miser sur les secteurs où elle a un avantage compétitif qui pourront dans les années qui viennent créer des emplois et de la croissance et c'est l'objet précisément de ce grand emprunt

Le dernier segment de la question de JMA3, présentant le rattrapage énonciatif : « dit Libération», constitue la reprise de la première question de l'interview ${ }^{1}$ à laquelle $\mathrm{L}$. Chatel n'a pas répondu lors de sa première intervention. La réponse LCH4 commence par le début d'une réfutation du jugement médié rapporté (l'adverbe non) immédiatement avortée et suivie d'un glissement thématique : de la faillite de la France, dans la question médiée, à l'action gouvernementale face à la situation économique de la France (les trois fronts), ce qui revient à ne pas tenir compte de la médiation énonciative, quitte à répondre «à côté ». Le journaliste sera alors dans l'obligation d'interrompre l'interviewé pour reposer la question ${ }^{2}$.

\section{Invalidation de l'énonciateur}

Au lieu de répondre à la question que pose le jugement médié rapporté, la réponse contient un commentaire sur l'énonciateur rapporté, qui disqualifie son autorité :

(2) Interview de François Fillon par David Pujadas dans le JT de 20h de France 2, le 28 janvier 2009

DP5 - vous l'avez entendu monsieur le Premier ministre y a un sentiment pour beaucoup d'injustice / des milliards donnés aux banques qui sont considérées à tort ou à raison comme parties prenantes de la crise financière / et rien ou peu pour les Français pour la consommation pour le pouvoir d'achat

FF6 - y a un moment où il faut que chacun prenne ses responsabilités et je dirais y compris le système médiatique / on peut pas dire « à tort ou à raison donnés aux banques »/ pardonnez-moi mais je sais bien que c'est le discours qui est tenu comme ça par tout le monde

DP7 - c'est un sentiment qu'on entend et qu'on vient encore d'entendre...

FF8 - oui mais c'est pas une question de sentiment / qu'est-ce que diraient les Français si en octobre ils s'étaient présentés au guichet du Crédit Agricole du Crédit Lyonnais ou de la BNP et qu'on leur avait dit " le guichet est fermé vos économies se sont envolées »/ parce que c'est ça la situation face à laquelle le gouvernement s'est trouvé et donc ce que nous avons fait c'est sauver d'abord les économies des Français $[\ldots]$

Le journaliste interroge le Premier ministre à partir du jugement attribué à " un sentiment pour beaucoup» qui lui permet de légitimer son acte de parole, mais FF8 invalide la pertinence de ce « sentiment » après avoir attaqué, certes poliment, le journaliste au sujet de la responsabilité des médias dans le colportage de la rumeur populaire : «c'est le discours qui est tenu comme ça par tout le monde ». Le tour FF8 ne réfute pas ouvertement le propos rapporté, mais la crédibilité de l'énonciateur « tout le monde ». F. Fillon concède dans un premier temps l'argument avancé par l'autre oui, comme vous dites, c'est ce que l'on dit / il y a un sentiment, la concession est suivie d'un désaccord introduit par le connecteur mais : «mais ce n'est pas une question de sentiment», ce qui permet une réorientation argumentative. L'interviewé disqualifie l'actant « tout le monde» dans son statut d'énonciateur crédible 
et, du même coup, la pertinence de l'acte de parole rapporté se trouve remise en cause. L'écran énonciatif protecteur que place le journaliste, en convoquant le discours populaire, est déjoué par l'homme politique.

Dans l'exemple suivant, l'interviewé B. Tapie procède de manière plus grossière, en employant un registre de langue très familier, afin de disqualifier l'énonciateur du jugement rapporté, qui est dans ce cas un personnage politique connu :

(3) Interview de Bernard Tapie par Jean-Michel Aphatie dans RTL Matin, le 9 septembre 2008

JMA19 - vous aurez face à vous demain des adversaires très résolus Français Bayrou en fait partie / il a beaucoup critiqué cette sentence arbitrale et il a dit récemment « voilà un état qui n'a plus un sou dans ses caisses et qui donne le moyen à monsieur Tapie de rembourser ses dettes aux frais du contribuable »

BT20 - vous voulez pas en dire d'autres ? parce qu'il en a dit quelques-unes des conneries

JMA21 - il en a dit beaucoup...

BT22 - ouais ce que je voudrais vous dire c'est que je suis épaté moi / de l'écart qu'il y a entre l'importance que vous donnez à ce monsieur / où tous les jours on fait les commentaires de ce qu'il pense ce qu'il dit des bons points qu'il donne des mauvais points qu'il donne / alors que politiquement il incarne plus rien / il est à la rue politiquement / il a il a monté un mouvement qui s'appelle le Modem qui a deux députés il a pas été foutu de gagner les élections municipales chez lui / il a été battu entre parenthèses par une socialiste qu'il a traitée je vous dis pas comment avant de s'associer avec les socialistes / je pense qu'elle va avoir envie de raconter ses méthodes / et subitement on est là en train d'en faire le fer de lance de l'opposition

Le tour JMA19 est une question médiée relevant de la structure : thème, jugement médié rapporté, sans question explicite. L'intervention du journaliste sert à mettre en opposition le discours de l'interviewé et le discours attribué à un opposant exogroupal. L'interviewé évalue dans un premier temps (BT20) le propos rapporté comme «conneries» afin de l'invalider et, dans un second temps (BT22), il disqualifie son énonciateur. L'argument qui permet de contester l'autorité de l'énonciateur du jugement médié est, comme précédemment (2), l'absence de crédibilité : M. Bayrou raconte des conneries ( il en a dit quelques-unes des conneries ») donc il n'est pas un interlocuteur crédible (conclusion implicite). La réponse de Tapie débute de fait par une question négative « vous voulez pas en dire d'autres » qui va introduire l'enchaînement argumentatif minimal : argument correspondant à la proposition causale ( $\mathrm{p}$ ), suivi de la conclusion implicite (donc q). Le tour BT22 constitue un étayage argumentatif : «il (F. Bayrou) n'incarne plus rien, il est à la rue, le Modem a deux députés, il a été battu par une socialiste chez lui, ses méthodes sont louches...»

En invalidant l'autorité de l'énonciateur du jugement médié rapporté, l'interviewé remet en cause la pertinence de son jugement, ce que lui permet d'éviter de répondre à la question embarrassante.

\section{Rectification du jugement rapporté}

Définie de manière globale, cette stratégie de réponse consiste à faire un commentaire, le plus souvent critique, sur le contenu du jugement médié, plutôt que de répondre à la question sollicitée par celui-ci. La pertinence de la question de l'intervieweur se trouve invalidée du même coup, ce que l'on pourrait paraphraser de façon suivante : la question n'est pas valable parce que le jugement médié sur lequel elle est fondée n'est pas tout à fait exact. Nous pouvons distinguer plusieurs réalisations de cette stratégie : (i) l'interviewé reformule de façon critique le jugement, (ii) la reformulation du jugement est partielle et (iii) l'interviewé corrige le contenu du jugement rapporté.

\subsection{Reformulation critique du jugement}

La stratégie en question consiste, dans l'exemple analysé, à remplacer dans la réponse un terme employé dans le jugement médié par un autre, plus approprié selon l'interviewé, afin de dénommer le thème : 
(4) JMA23 - alors l'un des dossiers / c'est cette enquête confidentielle relevée par Armand Levy sur l'antenne de RTL ce matin / quarante-cinq pour cent des professeurs des écoles / donc maternelle et élémentaire / ont posé un congé maladie en 2007/2008 / c'est le double de ce qu'on constate dans le secteur privé / savez-vous / Luc Chatel / pourquoi les instituteurs sont plus malades que les autres ?

LCH24 - d'abord quand on parle d'absentéisme / il faut savoir de quoi on parle / c'est pas parce qu'un enseignant n'est pas devant un élève que son absence n'est pas justifiée et d'ailleurs tout à l'heure vous avez dit dans votre reportage / un enseignant il peut être en formation :: / il peut être en :: journée pédagogique / deuxième point $\mathrm{xxx}$

JMA25 - est-ce qu'il y a un problème d'absentéisme?

Le jugement médié porte sur le thème congés maladie des instits («c'est le double de ce qu'on constate dans le secteur privé »). Le journaliste interroge le ministre de l'éducation nationale fraîchement nommé sur les causes de ces congés, ce qui peut constituer un piège potentiel pour l'interviewé parce que (i) c'est un test de sa connaissance du dossier dont il vient de prendre possession et (ii) cela peut l'amener à évoquer les conditions de travail de plus en plus difficiles déplorées par les enseignants. La dénomination «congé maladie» se trouve reformulée dans la réponse en "absentéisme» (nom plus général, hyperthème à connotation négative, appartenant à l'univers du discours «l'absentéisme des professeurs »). Le sens de ce nom est glosé par la phrase clivée avec une double négation : ce n'est pas $X$ $q u$ - non $Z$. Le clivage s'oppose explicitement à un discours évaluatif répandu sur l'absentéisme des professeurs qu'il corrige partiellement, ce que l'on pourrait paraphraser comme suit : contrairement à ce que dit $X^{3}$ (les profs sont absents de façon abusive) il y a des absences justifiées. Le sens attribué au mot «absentéisme» par l'emploi du clivage est contraire à celui que l'on trouve habituellement dans un dictionnaire « absence d'un salarié de son lieu de travail, non justifié par un motif légal ». L'interviewé ne répond pas directement à la question, posée de manière explicite, mais interagit avec le jugement médié rapporté, en opérant une reformulation critique de son contenu qui vise à poser le problème dans toute sa complexité, en employant un terme plus englobant et de ce fait plus adéquat pour être le support d'un jugement. Cette stratégie rend la réponse assez ambivalente car (i) l'interviewé emploie un mot à connotation négative, notamment pour les professeurs, qui n'est pas le terme et le thème exact dans la question et (ii) il le fait dans le but de défendre les enseignants (l'emploi de la phrase clivée). L'homme politique cherche en somme un double consensus : à la fois avec l'opinion publique, en reprenant les objets communément partagés, et avec les professeurs qu'il semble défendre, même si le terme absentéisme peut au départ être perçu comme offensant pour eux.

\subsection{Reformulation partielle}

Cette stratégie est employée plus particulièrement lorsque l'interviewé est confronté au jugement endogroupal ou à l'autojugement, sur le principe : on ne remet pas totalement en cause le discours de son parti ou son propre discours :

(5) Interview de B. Hamon par Françoise Laborde dans Les quatre vérités, le 15 décembre 2008

FL15 - vous avez proposé vous-même quelque chose qui est de l'ordre du retour à l'autorisation administrative du licenciement // ça a un peu fait tousser / pour dire des choses simplement

BH16 - ça a fait tousser Monsieur Chatel notamment

FL 17 - oui mais ça a même fait tousser à gauche / il y a certains au PS qui ont dit « oh lala ! on va pas revenir à l'ancien système : :

BH 18 - on est tous d'accord sur une chose

FL19 - qui marche pas / parce que si ça avait marché on se serait rendu compte »

BH20 - je vais vous dire ce qui peut marcher très bien / c'est des entreprises qui gagnent de l'argent // aujourd'hui/ comment les empêcher de licencier alors qu'elles le font //ça existe ça il y en a plusieurs dizaines dans ce pays et elles mettent plusieurs milliers de français sur les carreaux / comment les 
empêcher mais c'est de ça qu'on parle au PS aujourd'hui et dont ne parle pas le gouvernement / c'est d'être en capacité aujourd'hui de dissuader financièrement une entreprise qui licencie/

La question médiée FL17 a la forme : thème, jugement médié rapporté, mais sans interrogation explicite. La journaliste essaie de piéger l'interviewé en mettant en contradiction son discours avec celui de son propre parti : «ça a même fait tousser à gauche... ». La réponse reformule le jugement partiellement, en insistant sur le consensus : « on est tous d'accord sur une chose » qui se poursuit par la phrase clivée en BH20 «c'est de ça qu'on parle au $\mathrm{PS}^{4}$ » dans laquelle le pronom démonstratif ça est cataphorique de l'énoncé final de l'intervention de BH («c'est d'être en capacité aujourd'hui de dissuader financièrement une entreprise qui licencie »), de manière à minimiser le dissensus que faisait apparaître la question. La réponse ne remet pas totalement en cause l'existence dudit dissensus, mais fait apparaître, au premier plan, un autre aspect relatif à l'objet du discours : ce qui est partagé, afin de modaliser le jugement rapporté, car ce qui est partagé (comment empêcher les entreprises qui marchent bien de licencier) est plus important que ce qui divise (l'autorisation administrative de licenciement proposée par BH).

\subsection{Rectification de l'acte de parole rapporté}

La rectification consiste à corriger le contenu de l'acte de parole rapporté qui n'est pas conforme à sa source originale, selon l'interviewé. Elle s'opère préférentiellement lorsqu'il s'agit de corriger le propos prêté par l'intervieweur à l'interviewé qui, parce qu'il a participé à l'interaction rapportée, occupe le rôle discursif du témoin du vu / entendu qui peut attester de l'exactitude ou de l'inexactitude du jugement rapporté :

(6) JMA11 - le Figaro ce matin raconte que vous Luc Chatel / nouveau ministre de l'éducation / vous avez dit hier en compagnie des autres membres du gouvernement / vous avez dit que vous auriez voulu profiter / vous voudriez profiter de cet emprunt pour rénover les écoles / et le premier ministre vous a dit pas question

LCH12 - non / moi j'ai évoqué plusieurs secteurs / vous savez c'était une réunion brain storming / où chaque ministre évoquait un certain nombre de pistes pour accompagner la sortie de crise et tout naturellement moi j'ai évoqué l'école du futur à savoir le numérique (....) donc nous avons eu une réunion mais $\mathrm{xxx}$

JMA13 - mais le Premier ministre François Fillon vous a dit non?

Le tour LCH12 rectifie l'acte de parole rapporté, en l'occurrence le sien, par l'emploi de la négation (afin de rejeter l'exactitude de l'acte de parole rapporté), suivie de l'insistance pronominale (« moi, je », afin de légitimer son statut de source) et de la mention du propos exact. Il enchaîne, dans la suite de la réponse, par un commentaire sur les circonstances (la réunion) dans lesquelles ledit propos a été tenu.

\section{Recadrage de l'interaction rapportée}

\subsection{Différer l'interaction}

La réponse contient des commentaires sur les circonstances spatio-temporelles d'énonciation du jugement, sur lesquelles ne portait cependant pas la question :

(7) JMA23 - mais sur le fond ? il (François Bayrou) dit « l'état n'a plus de sous ...

BT24 - non mais sur le fond je suis heureux qu'il vienne demain pour tout vous dire

JMA 25 - et il va / et il va donner plusieurs centaines de millions xx à Bernard Tapie

BT26 - oui mais il va me le dire il va me le dire je l'espère demain quand je serai là / ce sera beaucoup plus facile / et ce sera surtout beaucoup plus pour moi rassurant de pouvoir dire aux gens la réalité / vous savez je m'attends à ce qu'un jour / comme il c'est sa fonction aujourd'hui / je m'attends à ce qu'un jour 
il déclare / « c'est un scandale qu'on meure encore du cancer » / et vous avez des gens qui diront « oui c'est vrai putain c'est quand même dommage qu'on meure d'un cancer »/ par conséquent mélangez pas / la mission d'information qui est un devoir normal et de l'assemblée de m'entendre et moi de parler / et les a-attributs que se donne monsieur Bayrou d'une espèce d'ordre nouveau dans lequel il a retrouvé d'ailleurs pour ce tandem fabuleux monsieur xxx c'est un très beau couple

Le premier tour de parole BT24 interagit non pas avec le contenu de l'acte de parole rapporté, mais avec les circonstances temporelles de son énonciation, en différant l'interaction avec l'énonciateur du discours rapporté à demain. BT26 réitère la même stratégie d'évitement : "il va me le dire demain, ce sera beaucoup plus facile ", ce qui permet à l'interviewé (i) de différer l'interaction avec l'énonciateur du jugement médié dans le temps et (ii) de refuser la médiation : l'interaction est différée au lendemain et dans la situation de face à face. Le refus de la médiation qui se termine en leçon de journalisme est une façon grossière d'invalider l'autorité journalistique ${ }^{5}$.

\subsection{Temporisation}

\subsubsection{Arrêt de jeu}

L'interviewé demande du temps pour la réflexion avant de répondre :

(8) Interview de J-F. Copé par O. Galzi, dans Les quatre vérités, le 9 décembre 2008

OG24 - autre sujet / le sénateur Philippe Marini qui fait décidément beaucoup parler de lui en ce moment propose de supprimer la demi part fiscale accordée aux parents quand ils n'ont plus leur enfant à charge / vous en pensez quoi?

JFC25 - bon écoutez là pour l'instant / je ne peux pas vous répondre parce que je n'ai pas vu en détail cette disposition / elle me paraît un peu surprenante / parce que si je vois bien qu'il y a derrière une idée d'économie euh :::

La réponse est remise à plus tard, sans que l'interviewé refuse la médiation journalistique. L'arrêt provisoire de l'interaction est justifié par la méconnaissance prétendue du dossier au moment de l'interview.

\subsubsection{Renvoi de la balle}

L'interviewé renvoie la question à l'énonciateur du jugement médié, " posez lui la question » :

(9) Interview de X. Bertrand par R. Sicard, dans Les quatre vérités, le 2 décembre 2008

RS 15 - mai::s pourquoi selon vous Laurence Parisot a dit ça ${ }^{6} / c^{\prime}$ 'est parce qu'elle pense que les résultats du Medef ne seront pas très bons?

XB 16 - posez lui la question / mais ce que je sais / j'étais tout à l'heure dans une entreprise en région parisienne / une entreprise qui s'est mobilisée / qui a informé ses salariés qui leur a donné aussi les moyens de pouvoir voter / (...)

L'interviewé se place en position de celui qui n'est pas le bon destinataire de la question, qu'il « réexpédie » à l'énonciateur du jugement médié rapporté, auquel elle devrait être posée lors d'une interview future.

\subsection{Légitimation du cadre situationnel}

L'interviewé dans sa réponse évite de traiter la question de l'intervieweur en s'attachant à légitimer le cadre situationnel de l'interaction au cours de laquelle a été tenu le propos, d'où procède la question : 
(10) JMA1 - Emprunt Sarkozy vous en avez parlé hier à Matignon où le gouvernement était réuni au grand complet / question du journal Libération à la une ce matin (JMA montre la une de Libération à LCH) « Emprunt Sarkozy : La France est-elle en faillite ? »

$\mathrm{LCH} 2$ : écoutez c'est quand même légitime que après le discours du président de la république au congrès :::/ après le remaniement du gouvernement / le premier ministre réunisse l'ensemble de son gouvernement pour mettre en perspective la feuille de route du président parmi laquelle figure effectivement la mise en place d'un grand emprunt pour investir dans ce qu'on appelle les secteurs de la sortie de crise

La question médiée correspond à la structure thème, interrogation rapportée, qui vaut pour les éléments (ii) + (iii) + (iv) du module présenté, en se substituant à la question du journaliste, qui lui fait écho. $\mathrm{LCH} 2$, au lieu de répondre à l'interrogation rapportée, produit un commentaire légitimant le contexte de l'interaction (la réunion du gouvernement au grand complet à Matignon), comme si c'était le contexte qui avait suscité la question et pas le thème (emprunt Sarkozy).

La stratégie du recadrage dans la réponse de l'interviewé consiste à jouer de la situation d'énonciation du jugement rapporté : (i) en différant l'interaction dans le temps et dans l'espace, (ii) en prenant le temps, afin d'approfondir la connaissance d'un fait et, par souci de crédibilité, d'éviter de répondre hâtivement, (iii) en renvoyant la question à la bonne personne, qui correspond dans ce cas à l'énonciateur du jugement rapporté et (iv) en faisant semblant de croire que la (mise en) question porte sur le cadre situationnel ce qui conduit à le légitimer.

\section{Recadrage de la question qui procède du jugement médié}

\subsection{Déplacer/Remplacer la question}

L'interviewé reformule de façon critique la question du journaliste :

(11) JMA15 - Gérard Aschieri secrétaire général de l'EFSU à votre propos / puisque vous êtes le ministre de l'éducation depuis mardi soir / c'est quelqu'un/ il parle de vous/ qui jusqu'ici n'a jamais manifesté de l'intérêt pour l'éducation nationale

LCH16 - bah parce que sans doute Monsieur Aschieri me connaît mal / et je vais remédier à ça / puisque je vais le rencontrer après-demain // donc vous voyez // vous savez ça fait plusieurs années que je suis parlementaire ::/ puis le membre du gouvernement / l'éducation ça concerne tous les français/ chacun

JMA17 - mais vous êtes intéressé ?

LCH18 - chacun de nos concitoyens

JMA19 - mais vous êtes intéressé ?

LCH20 - je suis intéressé d'abord en tant que père de famille / je suis intéressé parce que / un pays qui croit en son avenir / c'est un pays qui croit en l'avenir des ses enfants et donc en son éducation

JMA21 - mais vous vous êtes déjà penché sur un dossier de l'éducation nationale ? xxx

LCH22 - écoutez / il vous a pas échappé que j’ai été nommé par le président de la République et le Premier ministre ministre de l'éducation / donc oui je me suis penché sur les dossiers / simplement encore une fois ce qui est important c'est que / un pays / et le président de la République l'a rappelé dans son discours à Versailles lundi dernier / qui croit en son avenir / c'est un pays qui mise sur l'éducation nationale et c'est la mission qu'il m'a confiée /

Le tour LCH22 emploie la phrase pseudo-clivée (ce qu-z, c'est y) afin de déplacer la question suscitée par le jugement médié rapporté en JMA15. On remarquera à ce propos que la question JMA21 n'est pas médiée à proprement parler: elle prend le relais de la question médiée JMA15, tout comme les interventions précédentes JMA17 et 19. La réponse de LCH se déroule en deux temps : (i) l'interviewé répond très brièvement à la question du journaliste, puis, (ii), afin d'éviter d'entrer dans les détails, pour, 
par exemple, ne pas mentionner les noms des dossiers sur lesquels «il s'est penché », il déplace la question à l'aide de la phrase pseudo-clivée : « ce qui est important, c'est un pays qui mise sur l'éducation nationale ». La phrase tronquée ce qu-z de la pseudo-clivée constitue le thème, que l'on définit comme $c e$ dont on parle, ce qui est connu et partagé par les protagonistes de l'échange. En désignant comme thème la proposition « ce qui est important », l'interviewé déplace l'objet de la question initiale (JMA15, 17, 19 et 21 qui portaient sur l'intérêt de l'interviewé pour l'Education nationale) vers un propos général de l'importance des choses. La phrase pseudo-clivée corrige partiellement la question, elle sous-entend la négation : ce qui est important, ce n'est pas mon intérêt pour l'éducation, c'est que le pays mise sur l'éducation. D'autres structures du même type peuvent également être employées, afin de remplacer la question posée : « la vraie question, ce n'est X est Y», «ce n'est pas la question, la question c'est », « la question n'est pas là », etc.

\subsection{Retournement de la question}

Contrairement au renvoi de la question à l'instance d'énonciation du jugement rapporté, étudié en 3.2.2, dans le cas présent, l'interviewé renvoie la question au journaliste :

(12) RS22 - sur l'UMP / le secrétaire général Patrick Devedjian a dit que sa mission était accomplie / euh est-ce que c'est un poste / patron de l'UMP / qui vous intéresse ?

XB23 - il a dit qu'elle était accomplie / il a pas dit terminée / il a pas dit finie // et je vais vous faire une confidence / je suis secrétaire général adjoint de l'UMP / eh bien c'est très bien /

RS24 - mais vous avez pas l'intention de devenir secrétaire général tout court ?

XB25 - je vais vous dire en plus une chose / secrétaire général adjoint c'est très bien et le ministre du travail que je suis a du travail / on en a parlé tout à l'heure avec les prud'homales

RS26 - mais vous ne voulez pas me répondre secrétaire général...

XB27 - mais je viens de vous le dire / Patrick Devedjian est en fonction et ce n'est pas le sujet / ce n'est pas la question

RS 8 - sur le plan de relance (2) proposé par Nicolas Sarkozy /

XB29 - xxxx William xxxx est-ce que vous vous avez envie de prendre la place de William ${ }^{7}$ ?

RS30 - oh pas du tout

WE31 - oh je peux vous dire il est pas le seul!

$\mathrm{XB} 32$ - je sais pas si ça le rassure

Le retournement de la question XB29 est précédé en XB23 d'une rectification « Patrick Devedjian est en fonction », suivie du remplacement de la question XB27 : «et ce n'est pas le sujet / ce n'est pas la question ». XB29 justifie le déplacement de la question en retournant à l'intervieweur, tel un écho, le même type de question que celle qui vient de lui être posée, afin de démontrer son caractère incongru.

\subsection{Rentrer dans la question pour la déjouer}

L'interviewé fait semblant de jouer le jeu de la question/réponse, de rentrer dans la question, en répondant à celle-ci, dans le sens voulu par le journaliste, mais il le fait dans le dessin de ridiculiser la question :

(13) JMA35- François Hollande premier secrétaire du parti socialiste " Chacun connaît les relations de Bernard Tapie à Nicolas Sarkozy il faut savoir si cette relation a pu jouer pour obtenir la sentence arbitrale $\mathrm{xx}^{\prime \prime} .$.

BT36 - il est très fort s'il connaît les relations que j'ai avec le président franchement / parce que elles sont pas du tout ce qu'on en fait croire / ensuite euh la sentence a pas été rendue par monsieur Sarkozy / 
figurez-vous / ouais imaginons le pire voilà Sarkozy est mon meilleur ami voilà je suis Clavier voyez je suis Clavier

JMA37 - mais vous êtes ami avec Nicolas Sarkozy

BT38 - non malheureusement / parce que j'aurais bien aimé mais c'est pas le cas

JMA39 - Xxxx moment vous avez pu dire que...

BT40 - j'ai dit que j'étais ami ? jamais vous avez pu entendre ça parce que c'est pas vrai...

JMA41 - que vous aviez une relation ::: privilégiée peut-être avec lui ?

BT42 - ouais et pourquoi pas très privilégiée et amicale / ouais vous en remettez un peu / non je ne suis pas ami et pour tout et pour vous rassurer / faire bien comprendre que je me défile pas / je le regrette j'aimerais bien être son ami c'est pas le cas / mais imaginons le pire il est ami comment peut-il intervenir/

L'extrait présente deux réponses BT38 et BT42 qui procèdent de stratégies relativement proches basées sur l'ironie. Le tour de parole BT38 est en interaction avec JMA37 qui constitue la reprise de la question médiée JMA35. BT38 présente un phénomène relevant de l'ironie lié au fait que l'interviewé, au lieu de réitérer la réfutation exprimée précédemment en BT36 (les relations de BT avec le président ne sont pas du tout ce que l'on fait croire), exprime son regret de ne pas pouvoir répondre par la confirmation à la question posée : «non malheureusement, parce que j'aurais bien aimé ». La réponse de l'interviewé pointe le décalage entre le désir malheureux (de l'autre : obtenir un aveu et de soi-même) et la réalité, faisant entendre l'allusion au dicton populaire : ne prenez pas vos désirs pour les réalités. En affirmant ouvertement sa préférence, la réponse de BT est censée être un gage de sincérité, face à la mauvaise foi du journaliste qui essaie de "forcer le trait", "d'insinuer", "d'accuser à tort", en se réfugiant derrière l'écran protecteur de la médiation énonciative.

BT42 débute par la confirmation (« ouais »), dans la mesure où la question du journaliste, non satisfait de la réponse, est renouvelée. La confirmation est ce que le journaliste veut entendre, mais ne s'attend pas à entendre. Elle est immédiatement suivie d'une autre question qui fait écho à celle du journaliste, en renchérissant par l'addition de l'adverbe très à la reprise du SA privilégiée du discours de l'autre. C'est une manière de dénoncer ironiquement le fait que la question n'aille pas suffisamment loin dans la révélation. BT42, en confirmant, fait semblant de jouer le jeu. En renchérissant, il se moque de la question du journaliste, la tourne au ridicule, sans y répondre franchement. Dans la suite de la réponse, l'interviewé réitère la réfutation d'une part, et d'autre part l'expression de son regret, une façon de prouver sa bonne foi.

\section{Du posé au présupposé : exploitation du contenu implicite}

Dans tous les cas présentés jusqu'ici, les stratégies de l'interviewé exploitaient le posé de l'énoncé journalistique. L'interviewé était soumis à la question proposée à la mise en débat. Mais cela n'est pas toujours le cas, parfois la stratégie consiste à interagir avec le contenu implicite, inhérent à la question. Cette interaction peut prendre différentes formes : invalider la conclusion implicite, invalider le bien fondé de la question, recycler un argument négatif en positif

\subsection{Invalider la conclusion implicite de la question}

Soit l'exemple suivant :

(14) DP9 - un mot sur cette grève / il a beaucoup été question ces derniers jours / et notamment avec ce qui s'est passé à Saint-Lazare / de réaménager la loi sur le service minimum qui ne donnait pas dit-on satisfaction / est-ce que vous êtes pour? est-ce qu'il y a un calendrier?

FF10 - alors d'abord la loi sur le service minimum elle fonctionne elle s'applique et on va la voir s'appliquer jeudi c'est-à-dire que l'on peut déjà dire que jeudi y aura une offre de service public y aura 
une offre de transports en particulier qui sera nettement supérieure à ce qui se serait produit s'y avait pas la loi sur le service minimum $[\ldots]$

Dans la question médiée, la modalisation en discours second est intégrée dans une relative : « la loi sur le service minimum qui ne donnait pas dit-on satisfaction / " : certes, le jugement " qui ne donnait pas satisfaction » est explicitement référé à un énonciateur autre, par l'incise dit-on. Cependant, d'être enchâssée dans une relative appositive, cette formulation tend à être soustraite de l'interaction - son contenu est enfoui syntaxiquement - notamment de l'évaluation de l'homme politique, et peut passer pour « vraie " : même si elle est référée énonciativement à un autre énonciateur (on), le locuteur semble la prendre à son compte et implicitement penser que l'interlocuteur en fait de même, notamment du fait du temps du procès, l'imparfait ${ }^{8}$ (donnait).

Parce qu'elle présuppose que la loi sur le service minimum ne fonctionne pas, la formulation de la question est un piège potentiel pour le politique, piège dans lequel F. Fillon ne tombe pas, car, dans sa réponse, il commence par réfuter le présupposé. Le connecteur d'intégration linéaire «d'abord » est suivi de la dislocation à gauche du SN «la loi », repris par le pronom « elle» sujet du prédicat (fonctionne et s'applique), comportant le jugement positif qui invalide la conclusion implicite (la loi ne fonctionne pas), inférable de la relative appositive dans la question.

\subsection{Invalider le bien-fondé de la question en invalidant la présupposition dont elle procède}

L'exemple suivant exploite le contenu implicite de manière différente : LCH6 réfute la question en justifiant la réfutation par l'invalidation de la présupposition :

(15) JMA1 - Emprunt Sarkozy vous en avez parlé hier à Matignon où le gouvernement était réuni au grand complet question du journal libération à la une ce matin : Emprunt Sarkozy : La France est-elle en faillite?

$[\ldots]$

JMA3 - parce qu'il n'y a plus de sous dans les caisses ? parce que la France est en faillite ? dit Libération.

$[\ldots]$

JMA5 - et alors la France est-elle en faillite ? oui ou non

LCH6 - la France n'est pas en faillite parce qu'un état n'est pas en faillite Jean-Michel Aphatie / la France elle est dans une situation budgétaire que l'on connaît tous hein à savoir que en gros les recettes couvrent environ deux tiers de nos dépenses

La question à laquelle répond l'intervention de LCH6 n'est pas dans sa formulation une question médiée, toutefois elle reprend les deux questions médiées qui la précèdent. Le journaliste exaspéré par la langue de bois lance un ultimatum à l'invité politique ${ }^{9}$. La réponse invalide la présupposition sur laquelle s'appuie la question (un état, comme la France, peut être en faillite). L'invalidation correspond au syllogisme suivant : un état n'est pas en faillite (la majeure), la France est un état (la mineure) donc elle n'est pas en faillite. Ce raisonnement remet en cause le bien fondé de la question, à condition d'admettre les deux propositions : la majeure et la mineure, sur lesquelles s'appuie la conclusion du syllogisme.

\subsection{Recycler un argument négatif en positif}

Les arguments négatifs pour l'interviewé, dans la question du journaliste, sont recyclés en positifs, dans la réponse, en exploitant le contenu implicite :

(16) OG11 - enfin y a (2) aussi y a aussi les députés de droite qui grognent un petit peu//François Baroin, par exemple chez vous dit cette réforme de la gouvernance de France télévisions/ nommer le président de 
France télévisions / le faire nommer par le président de la République / c'est contre la démocratie, enfin c'est presque un recul démocratique et il est UMP.

JFC12 - encore une fois attendez / moi je vous dis moi vous savez je suis responsable de la majorité UMP/ je suis donc garant de la liberté d'expression / chacun a toute liberté de dire ce qu'il pense et heureusement/ on est en deux mille huit / heureusement que dans mon groupe mes trois cents vingt amis députés ont chacun leur sensibilité / ce que je dis simplement / c'est que entre exprimer une opinion et bloquer le parlement pour un texte comme celui qui prévoit une télévision publique puissante et modernisée / c'est juste avoir la tête à l'envers / ce que je dis aux socialistes / c'est que moi je respecte toutes les oppositions mais bloquer le parlement pour ça / c'est hallucinant // [...] / c'est juste n'importe quoi/

Au lieu de répondre à la question que pose la médiation embarrassante, l'intervention de J-F. Copé débute par la légitimation de l'énonciateur au nom de la liberté d'expression. La proposition «chacun a toute liberté de dire ce qu'il pense ", évaluée par l'adverbe d'énonciation heureusement, devient un argument d'autorité dans la mesure où l'interviewé reprend à son compte l'assertion d'un autre, d'un on de vérité générale : comme chacun le sait, selon le principe général, la démocratie garantit la liberté d'expression, pour en faire «le reflet de la réalité » (Ducrot $1984: 168$ ). La question médiée contient des arguments négatifs pour l'interviewé : le discours narrativisé «les députés de droite grognent un petit peu (qui sous-entend que la réforme divise le groupe UMP), le jugement médié rapporté défavorable « c'est contre la démocratie ", conduisant à la conclusion implicite que la réforme n'est peut-être pas bonne. Dans la réponse, l'interviewé recycle ces arguments négatifs en arguments qui deviennent positifs, grâce au syllogisme : la démocratie garantit la libre expression des points de vue (argument positif = la majeure : " chacun a toute liberté de dire ce qu'il pense »), à l'UMP chacun a le droit de s'exprimer librement (la mineure) donc le groupe UMP est démocratique (conclusion implicite positive pour l'interviewé qui en est le garant en tant que son président : « je suis donc garant de la liberté d'expression »). La conclusion implicite assure la cohérence avec la suite de la réponse, syntaxiquement très complexe. L'interviewé y développe (i) un recadrage de son propre propos à l'aide d'une succession de deux phrases pseudo-clivées ce que je dis, c'est que, et (ii) la comparaison avec la conduite antidémocratique des socialistes, les pseudo-clivées se combinant avec la dislocation à gauche d'un syntagme repris par le pronom ce du présentatif c'est : «bloquer le parlement, c'est juste avoir la tête à l'envers », pour la première, et avec l'insistance pronominale moi/je + la dislocation du même type que la précédente «bloquer le parlement pour ça, c'est hallucinant», pour la seconde. La complexité syntaxique est porteuse d'une double thématisation. La phrase tronquée du pseudo-clivage (ce que je dis) désigne comme thème le propos antérieur de l'interviewé qui va être reformulé. La dislocation thématise l'élément disloqué à gauche afin de permettre la comparaison entre l'interviewé ainsi que son groupe et l'opposition, comparaison défavorable à cette dernière. La question de la réforme de l'audiovisuel se trouve ainsi diluée dans le recyclage argumentatif enfoui sous une complexité syntaxique.

\section{Conclusion}

La médiation énonciative offre au journaliste l'écran protecteur qui lui permet d'interroger l'invité politique sur les points sensibles, de poser des questions intrusives et embarrassantes, sans s'exposer ouvertement aux contre-attaques du politique. Ce dernier, poussé dans ses retranchements, doit éviter de tomber dans le piège que pose la médiation, en usant de différentes stratégies afin de contourner, voire d'évacuer, la difficulté qu'elle pose. L'écran protecteur employé par le journaliste s'avère plus ou moins étanche, et se trouve le plus souvent déjoué par l'interviewé. L'étude de notre corpus d'interviews politiques a permis de distinguer et de décrire cinq catégories de stratégies de réponse à une question médiée (ou non médiée dans sa formulation, mais employée en relais de celle-ci, lorsque le journaliste n'obtenait pas de réponse satisfaisante du premier, voire du deuxième coup). Les stratégies sont basées sur le traitement par l'interviewé de l'un des paramètres de la médiation énonciative : 1) l'énonciateur de l'acte de parole rapporté, afin de le disqualifier, 2) le contenu de l'acte de parole rapporté, afin de le rectifier de différentes manières, 3 ) le cadre situationnel dans lequel l'acte de parole a été énoncé, afin de 
le recadrer, 4) la question du journaliste procédant de la médiation embarrassante, afin de la rectifier, 5) le contenu implicite, afin de produire une réorientation argumentative favorable à l'interviewé.

La question médiée, en apparence plus difficile pour l'interviewé, dans la mesure où elle prend appui sur un dit antérieur polémique, permet de fait à l'homme politique d'exploiter habilement la structure de l'interaction d'où procède le propos rapporté, en faisant montre de sa capacité à retourner l'interaction à son avantage.

\section{Références bibliographiques}

Bres, J., (2008). De l'épaisseur du discours : horizontalement, verticalement... et dans tous les sens. Actes du premier Congrès mondial de linguistique française, http://www.ilf-cnrs.fr/

Bres, J. et Nowakowska, A., (2006). Dialogisme : du principe à la matérialité discursive. In Perrin L. (éd.), Le sens et ses voix, Recherches linguistiques 28, Metz : Université de Metz, 21-48.

Burger, M. (2004). Média Debates: the Discours of the Host in a TV Talk-Show. In Carlos Gouveia, Carmida Silvestre \& Lu'sa Azuaga (ed), Discourse, Communication and the Enterprise: Linguistics Perspectives, Lisbon : Ulices, 345-357.

Burger, M. \& Martel, G., (2005). Argumentation et communication dans les médias. Québec : Nota Bene.

Charaudeau, P., (2005). Le discours politique. Les masques du pouvoir. Paris : Vuibert.

Clayman, S., Elliott, M., Heritage, J. and McDonald, L. (2006). Historical Trends in Questioning Presidents, 19532000, Presidential Studies Quarterly 36(4): 561-583.

Clayman, S. \& Heritage, J., (2002). Questioning Presidents: Journalistic deference and adversarialness in the press conferences of Eisenhower and Reagan. Journal of Communication 52(4), 749-77.

Clayman S. \& Heritage J., (2002), The News Interview: Journalists and Public Figures on the Air, Cambridge : Cambridge University Press.

Constantin de Chanay, H. \& Kerbrat -Orecchioni, C., (2007). 100 minutes pour convaincre : l'éthos en action de Nicolas Sarkozy. In M. Broth et al. (éd) Le français parlé des médias, Stockholm, Acta Universitatis Stokhomiensis, 309-329.

Ducrot O., (1984). Le dire et le dit. Paris : Minuit.

Englebretson, R. (éd.), (2007), Stance taking in Discourse. Amsterdam : John Benjamins.

Fairclough N. (1995). Medias Discourse. London : Edward Arnold.

Fairclough N., (2003). Analysing Discourse : Textual Analysis for Social Research. London : Routledge.

Fairclough N. (co-ed), (2007). Discourse in Contemporary Social Change. Berne : Peter Lang.

Heritage J., 2002, «The Limits of Questioning: Negative Interrogatives and Hostile Question Content », Journal of Pragmatics, 2002, 34:1427-1446.

Kerbrat -Orecchioni C. (1996). «Vraies » et « fausses » questions : l'exemple de l'émission. Radiocom c'est vous. In J. Richard Zappelle (éd.), Le Questionnement social, IRED, université de Rouen, 37-45.

Kerbrat-Orecchioni, C. (1991). Introduction. In Kerbrat-Orecchioni C. (éd.), La question, Lyon : PUL, 5-37.

Nowakowska, A. \& Bres, J. (2009). La dimension dialogique dans un acte de langage dialogal : les questions du journaliste dans l'interview politique. Communication au Colloque international Le français parlé des médias : les médias et les politiques, du 1 au 4 septembre 2009, UNIL Lausanne.

\footnotetext{
${ }^{1}$ JMA1 : Emprunt Sarkozy vous en avez parlé hier à Matignon où le gouvernement était réuni au grand complet question du journal Libération à la une ce matin : Emprunt Sarkozy : La France est-elle en faillite ?

${ }^{2}$ JMA5 : et alors la France est-elle en faillite ? oui ou non
} 
${ }^{3}$ L'identité de l'énonciateur X à qui est imputé l'énoncé partiellement rejeté et corrigé peut être assimilée à la fois à l'enquête confidentielle rapportée à l'antenne et à l'opinion publique dont le discours est confirmé par l'enquête.

${ }^{4}$ La phrase clivée s'oppose, implicitement, la négation est sous-entendue, à la question de la journaliste qu'elle corrige partiellement : c'est de cela et pas simplement de ma proposition qu'on parle.

${ }^{5}$ On remarquera que la même stratégie peut aisément être employée pour répondre à une question non médiée, mais embarrassante pour l'interviewé :

JMA 77 - eh bé on va leur demander d'accord / et vous combien vous avez gagné ?

BT78 - ça ça vous regarde pas et j'ai pris l'engagement XX ...

JMA 79 - ah ben voyez y a des questions que vous voulez qu'on pose et d'autres xxx....

BT80 - nononon mais j'ai pas dit que j'allais pas le dire j'ai dit «ça vous regarde pas aujourd'hui » / et si vous me laissiez finir vous l'auriez entendu / vous l'entendrez demain /

JMA 81 - xxx demain

Comme précédemment l'interaction est différée dans le temps et dans l'espace (vous l'entendrez demain, lors de l'audition dont il est question), après un refus de la question du journaliste jugée trop intrusive (ça ça vous regarde pas).

${ }^{6}$ RS13 : Sur le désintérêt de cette élection / Laurence Parisot la présidente du Medef a une explication / elle dit que le responsable c'est vous / parce que la campagne a été organisée dans la plus grande pagaille?

${ }^{7}$ William Leymergie est l'animateur de Télématin, l'émission sur France 2 dans laquelle s'insère l'interview Les quatre vérités.

${ }^{8}$ On remarquera la différence qu'il y aurait si le verbe était au conditionnel : « la loi sur le service minimum qui ne donnerait pas dit-on satisfaction / » : emploi dit journalistique du conditionnel qui met à distance énonciative la vérité de l'énoncé.

${ }^{9}$ Celui-ci a déjà essayé d'éviter de répondre en légitimant le cadre situationnel de l'interaction au cours de laquelle a été tenu le jugement rapporté (3.3.). 